\title{
Circuit Design Tools for Exploratory Understanding
}

\author{
Evan Strasnick \\ Stanford University \\ Stanford, USA \\ estrasni@stanford.edu
}

\begin{abstract}
Effective circuit design and debugging require developing an intimate understanding of the behaviors of a complex system. In my work, I've distilled barriers to such understanding to three fundamental challenges of circuit design: transparency, malleability, and modelability. In turn, my research contributes tools that address these challenges through novel changes to the circuit design workflow: Pinpoint improves transparency and malleability in the debugging of printed circuit boards (PCBs) by augmenting board connections with automatic instrumentation and reconfigurable connectivity. Scanalog similarly improves transparency and malleability in prototyping by providing an interactively reprogrammable platform on which to design and tune fully instrumented mixedsignal circuits. My ongoing work addresses issues in modelability through tools that generate empirically-derived fault models and highlight causal relationships between components in a circuit. By evaluating these interactions, my research examines the role of exploratory understanding in circuit design, asking, "How can tools promote understanding of a circuit by facilitating exploration and reflection?"
\end{abstract}

\section{Author Keywords}

circuit; physical computing; design tools; debugging; PCB; prototyping

\section{CCS Concepts}

-Human-centered computing $\rightarrow$ Human computer interaction (HCI); $\bullet$ Hardware $\rightarrow$ Printed circuit boards; Hardware test;

\section{INTRODUCTION}

Circuits surround us in an increasingly interactive world. With the proliferation of intelligent, networked, and embedded systems, designers now produce more electronic devices than ever before [1], and students have myriad opportunities to learn the essentials of electronics in the wake of an ongoing maker movement [2]. The success of these efforts hinges on

\footnotetext{
Permission to make digital or hard copies of all or part of this work for personal or classroom use is granted without fee provided that copies are not made or distributed for profit or commercial advantage and that copies bear this notice and the full citation on the first page. Copyrights for components of this work owned by others than the author(s) must be honored. Abstracting with credit is permitted. To copy otherwise, or republish, to post on servers or to redistribute to lists, requires prior specific permission and/or a fee. Request permissions from permissions@ acm.org.

UIST '19 Adjunct, October 20-23, 2019, New Orleans, LA, USA

(C) 2019 Copyright held by the owner/author(s). Publication rights licensed to ACM. ISBN 978-1-4503-6817-9/19/10 . \$ $\$ 15.00$
}

DOI: https : //doi .org/10.1145/3332167.3356876

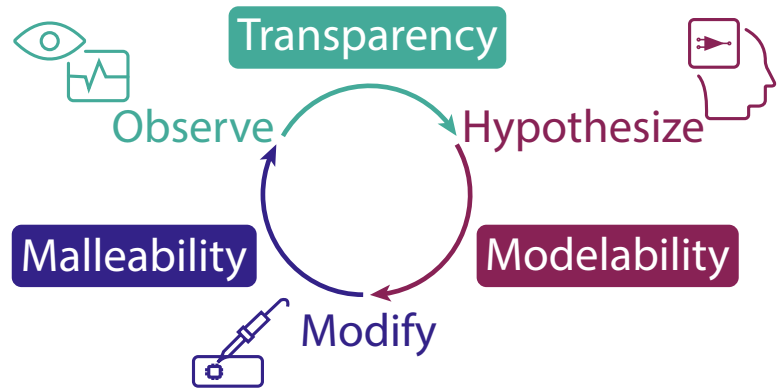

Figure 1. Circuit designers require efficient means of observing and modifying their circuit, as well as support to help form hypotheses in debugging. Correspondingly, transparency, malleability, and modelability are key properties for effective circuit design.

an ecosystem of tools enabling effective design and debugging of electronic devices.

However, in contrast to the diverse and evolving set of toolkits, environments, and debuggers that support software development practices, tools for hardware design have changed little over the past decades. The debugging of circuits remains a challenging, arcane practice, and in the course of my work, I have identified a set of fundamental qualities that facilitate a close coupling between designer and circuit. Unmet by current tools and common to both novice and expert applications, these core challenges (Figure 1) serve as goalposts to guide my research and to define directions for future tools:

1. Transparency. Designers must have the ability to readily observe behaviors throughout their circuit. Circuits are rife with hidden state information and afford limited access to our many observational tools, such as multimeters and oscilloscopes. As a result, designers face a large gulf of evaluation [3], in which simply inspecting circuit behaviors can be challenging.

2. Malleability. Circuit design is inherently iterative. Designers require the freedom to explore potential modifications and to temporarily reconfigure the circuit as needed to perform tests. However, for most forms of circuits, these operations range from manual, slow, and error-prone (in the case of the breadboard) to impossible without deconstructing the circuit (in the case of a PCB).

3. Modelability. Even with the means to observe and modify their circuit, debugging requires the construction of accurate conceptual models. Models enable the generation of 


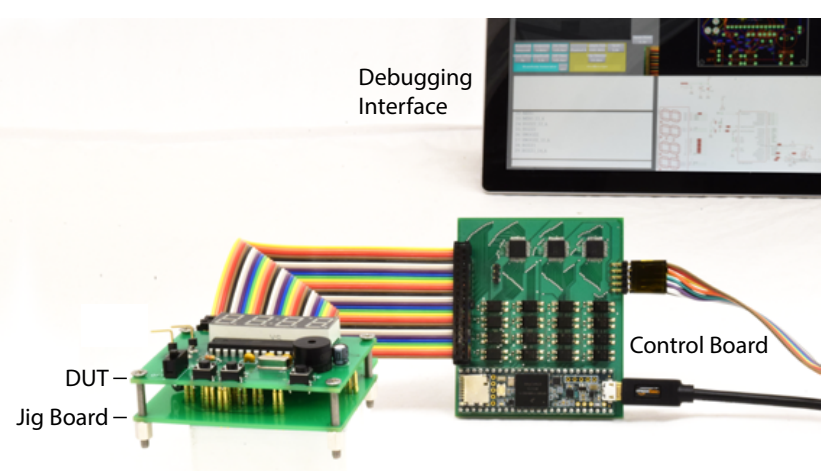

Figure 2. Pinpoint generates a custom hardware interface ("Jig Board") for a user's PCB ('DUT'), which connects to custom testing hardware ("Control Board"). As a result, the user can debug via a software interface with access to in-circuit testing features such as probing, signal injection, interruption of connectivity, and splicing in of external circuits.

hypotheses in response to observed errors, guiding the designer's decisions of which measurements to take and which alterations to make. For example, upon observing an unexpected output, a designer must trace the issue back up a chain of dependencies between components, defining a slice of the circuit in which a fault is likely to reside. However, the inter-component relationships that permit such tracing are often difficult to characterize, such as in analog circuits where networks of components behave as a system in equilibrium, rather than as a chain of inputs and outputs. Modelability enables designers to understand (e.g. causal) relationships between parts of their circuit, and how changes in the circuit might manifest as changes in behavior.

My work addresses these challenges through the development of novel tools and platforms that better support transparency, malleability, and modelability in circuit design. I utilize instrumented and programmable hardware to better support designers' ability to make exploratory perturbations to a live circuit and to draw insights from the response - thereby fostering what I refer to as exploratory understanding.

Two of the systems I have created target transparency and malleability: Pinpoint [6] by facilitating testing and iteration on PCBs, and Scanalog [5] by providing an interactively reprogrammable platform on which to design and tune mixedsignal circuits. My ongoing work then addresses the issue of modelability, using approaches including fault modeling and sensitivity analysis to support the generation of debugging hypotheses. Together, these interactions facilitate a new workflow in which designers can immediately observe and sensemake signals throughout their circuit, while interactively adjusting circuit parameters to reach the desired behavior.

\section{PINPOINT}

With benefits in robustness, compactness, and ease of mass production, PCBs are often considered the end stage in the refinement of a circuit design. In turn, they also present the largest challenges in transparency and malleability. Faults on a circuit board often lie hidden beneath small, surface mount components, making them challenging to identify even with sophisticated tools. As components on a PCB are mounted
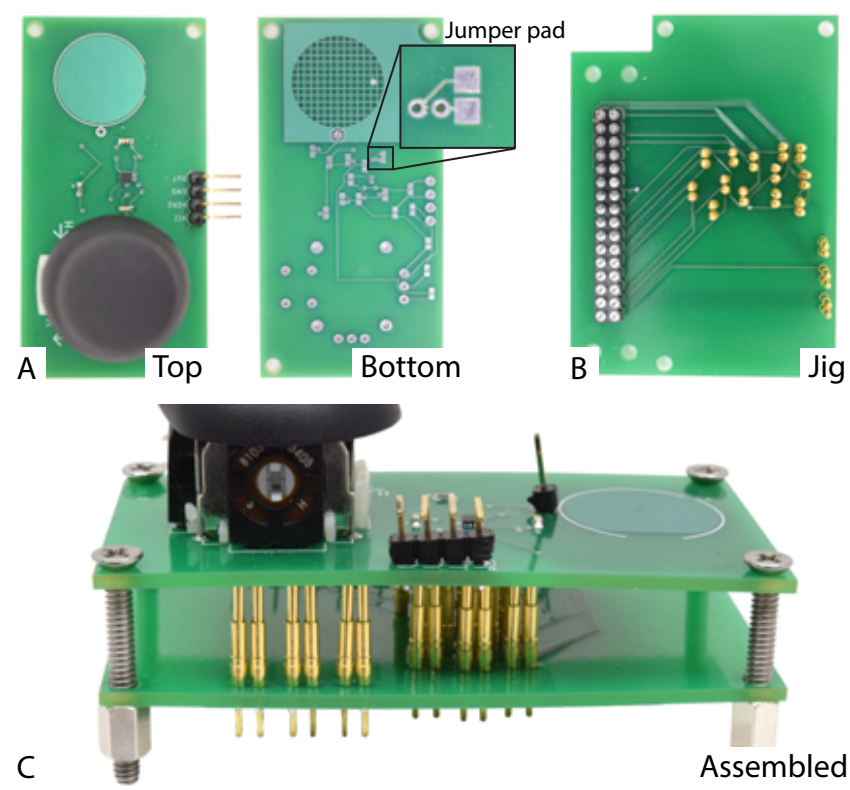

Figure 3. (A) The top and bottom (mirrored for clarity) of a PCB instrumented with Pinpoint. Pinpoint automatically introduces jumper pads (callout) in traces on the user's board. (B) The corresponding jig board generated by Pinpoint. (C) The jig board mates to the user's PCB with each pogo pin probing a pad.

with solder and connected with fixed traces, the designer cannot readily adjust the circuit for testing or iteration.

I address these challenges with Pinpoint (Figure 2), which provides a customized hardware-software interface for a given PCB design, enabling users to inspect, perform in circuit testing on, and even modify their board through software control. Pinpoint spans the entire PCB pipeline, from design to fabrication and debugging: It first automatically instruments all traces on a target PCB design with "jumper pads" — test pad pairs that can be programmatically connected or disconnected (Figure 3.A, callout). It then generates a design for a secondary "jig board" (Figure 3.B) that interfaces signals on the user's PCB to custom testing hardware ("control board") controlled by a graphical interface.

The resulting setup first allows Pinpoint to improve transparency by visualizing live signals from any point in the circuit. Further, by routing the user's traces across relays on the control board, Pinpoint can temporarily open connections or splice in new subcircuits to test potential solutions - improving malleability of PCBs in ways that current state-of-the-art test instruments cannot. These added affordances permit new approaches to in-circuit testing and integration testing: designers can isolate individual components (e.g. to check for faults), separate functional subcircuits (e.g. to isolate causes of noise), or even "stub-out" parts of a circuit with known-good hardware to focus on a particular section of the circuit.

\section{SCANALOG}

While Pinpoint address fundamental difficulties in debugging the many PCBs in our devices today, I anticipate that by exploring the use of programmable hardware, we can further 


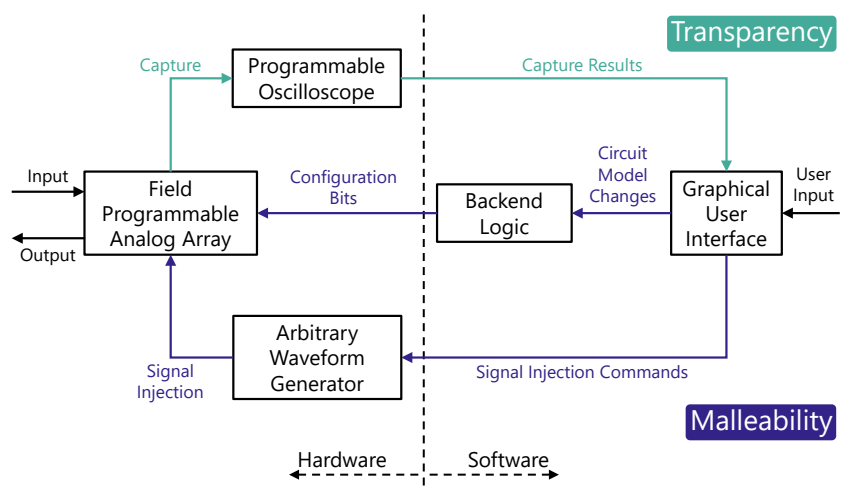

Figure 4. Functional diagram of Scanalog. Live instrumentation of all circuit elements promotes transparency, while real-time updating of programmable hardware offers malleability.

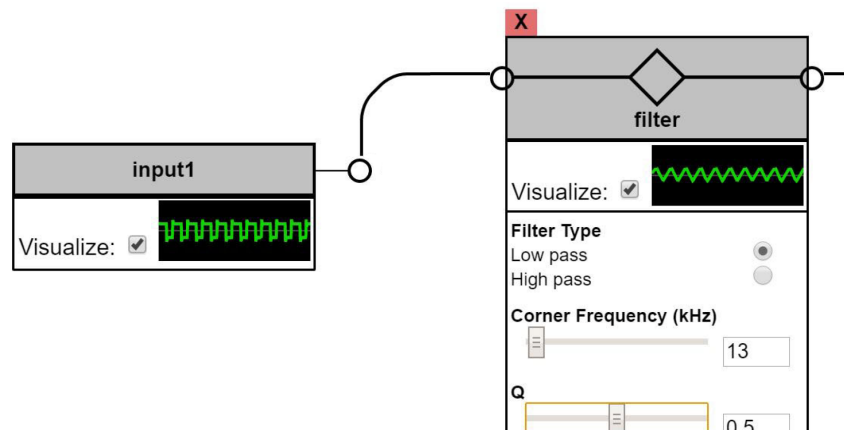

Figure 5. Configuring a filtering stage for a sensor in Scanalog's graphical interface. The designer can tune the filter parameters while observing the response throughout the circuit in real time.

accelerate debugging workflows to support transparency and malleability at a truly interactive pace.

For digital systems, Field Programmable Gate Array (FPGA) technology now plays an important role in reconfigurable hardware solutions, and ongoing research efforts seek to develop higher-level languages that can efficiently compile to FPGA logic [4]. In contrast, programmable technology for analog and mixed-signal systems is far less studied. Of particular interest is the Field Programmable Analog Array (FPAA), technology capable of programmatically configuring mixed-signal circuits using a bank of internal components and switchable routing. However, existing tools for configuring FPAAs fail to facilitate fluid iteration in the circuit design workflow: users still construct a complete schematic with carefully sized components, program the finished configuration, and manually test the output.

Scanlog extends the FPAA to utilize interactive reconfiguration. As the designer adjusts parameters of the circuit in a graphical interface, changes are immediately reflected in the underlying hardware. To maximize transparency, live signal responses from all points in the circuit are visualized alongside their schematic representation in real time (Figure 4). Instead of requiring the user to size and combine low-level components to achieve a target signal transform, Scanalog circuits are designed using high-level modules with parameters tunable through direct manipulation (e.g. gain, filter, subtraction). For example, a designer might add a low-pass filter to the output of a sensor, then scrub the filter parameters while observing the change in response to different stimuli (Figure 5). Since the working circuit is functional hardware, designers can take advantage of Scanalog's malleability to tune the circuit in-situ within the context of their application.

\section{ONGOING WORK}

My research to date has produced novel ways to inspect and modify live circuitry. In turn, my ongoing work targets outstanding aspects of the design process for which tool support remains lacking. This includes improving modelability by assisting in hypothesis generation, as well as integrating transparency, malleability, and modelability to evaluate their combined potential to enable more effective workflows for circuit design and debugging.

\section{Empirical Modeling of Inter-Component Relationships}

As with software, circuit debugging relies heavily upon the use of a conceptual model (implicit or explicit). Between the stages of observing an error and investigating a part of the circuit, the designer typically: 1) enumerates circuit elements on which the erroneous behavior might depend, 2) considers possible faults in the enumerated elements, 3 ) predicts how each fault might manifest as error and propagate throughout the circuit, and 4) devises corresponding experiments that can discriminate whether a given fault has occurred. In each step the conceptual model assists us in moving from observations to actionable debugging hypotheses. Key in these models is a notion of causality - by understanding the dependency relations between parts of a system, we learn how faults are likely to manifest, and we can plan experiments whose outcomes are useful in narrowing our hypotheses.

Within digital logic, such dependencies are typically straightforward: values cascade from inputs to outputs. But, particularly in mixed-signal and analog circuits, causal and feedbackcausal relationships become increasingly unclear as complexity grows. While designers may add a subcircuit known to perform a particular function (e.g. an amplification stage), the contribution of each individual component to the overall circuit response often requires exploration to understand. Without clear causal relationships (i.e. without modelability), it can be difficult to generate debugging hypotheses.

While currently there exists little direct tool support for modelability in circuits, simulation can aid in revealing the propagating effects of design changes. However, the majority of real-world devices are not easily simulated - modern integrated circuits are black-boxed components with complex internal designs and deviations from ideal behaviors. Rather than fashioning detailed simulations for each of the many components they utilize, designers instead gain insights through prototyping and testing. Therefore, there is need for a system that affords us the rapid modeling capabilities of simulations while utilizing empirical tests on real-world components.

Towards that goal, my current work establishes a system (Figure 6) for modeling dependencies within a live circuit by experimentally manipulating its parameters. In short, using programmable hardware we can rapidly inject changes (such 


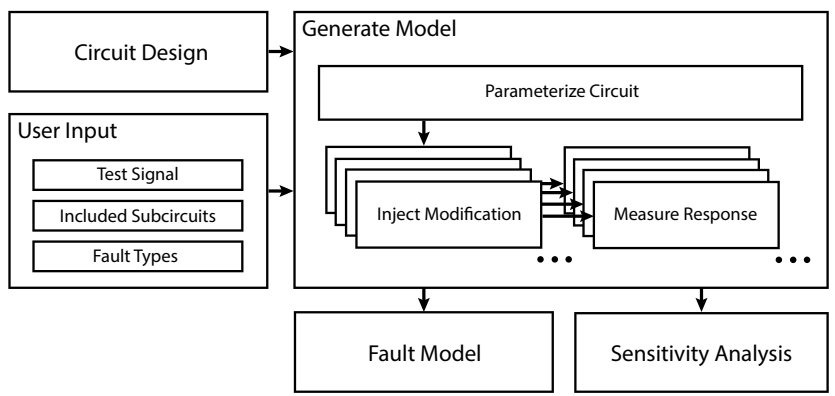

Figure 6. Empirically modeling dependencies within a circuit. The system enumerates parameters to manipulate on the target circuit, then injects modifications via programmable hardware and measures responses throughout the circuit against a test signal. The resulting model suggests possible faults given observations from the user's live circuit, and characterizes the sensitivity of relationships between components and signals.

as a short between signals or an increased value for a capacitor) into a real circuit and measure responses to a test input across all signals. In repeating this process, we develop a model of the effects of each change as we explore a parameter space of possible modifications. The system affords two main benefits:

1. It learns a fault model for a given circuit that supplies likely hypotheses for observed error behavior.

2. It performs a sensitivity analysis on elements in the circuit, visualizing the strengths of relationships between components and signals.

This tool therefore aims to improve modelability by supporting hypothesis generation during debugging: When the circuit produces an unexpected output, the tool can compare live measurements across the entire circuit to those within the precomputed fault model and suggest potential faults ordered by degree of match. Similarly, it can highlight components across the circuit that play significant roles in determining the value of a given output, providing the user with a relevant slice of the circuit to debug. In the inverse direction, these dependency models also afford feed-forward predictions to help guide exploratory design: when deciding to modify a given element, the user can anticipate which signals throughout the circuit are likely to be affected.

A number of ongoing challenges remain to be solved as I continue to design this tool: How do we parameterize a circuit in such a way that exploring the resulting parameter space remains tractable? How does the user supply design knowledge about the circuit to help constrain this space? How do we best visualize or present the resultant model to support effective debugging?

\section{Towards Test-Oriented Debugging Workflows}

Tools combining principles of transparency, malleability, and modelability can enable more powerful approaches to debugging than simple point measurements of voltage and current. Specifically, these properties jointly facilitate test-oriented workflows, in which users specify intended responses of their circuit to test cases and make use of automation to help identify issues. Test-oriented workflows offer numerous advantages over reactive, measurement-based debugging. For example, a well-designed test suite aids in sensemaking by associating errant behaviors with specific faults, and it facilitates fast iteration by quickly verifying previously debugged properties on a new version. Currently, both Pinpoint and Scanalog include features designed to promote test-oriented workflows, such as the ability to record and replay observed signals, decorate a circuit with live assertions, and construct suites of unit tests. Further facilitating test-oriented practices remains an ongoing goal of my research.

\section{CONCLUSION}

In my work, I've explored the design of tools built on instrumented, programmable circuit platforms to address fundamental challenges in circuit design and debugging by improving transparency, malleability, and modelability. These tools introduce novel approaches to debugging with a focus on exploratory understanding. My ongoing work addresses remaining challenges in hypothesis generation, as well as combines the novel interactions I have explored to promote more effective (e.g. test-oriented) workflows. Through these contributions, I aim to promote new paradigms for circuit design which more closely couple designer and circuit and facilitate iterative, exploratory practices.

\section{ACKNOWLEDGMENTS}

I thank my many collaborators and mentors for their continued support. This research was conducted with Government support under and awarded by DoD, Air Force Office of Scientific Research, National Defense Science and Engineering Graduate (NDSEG) Fellowship, 32 CFR 168a.

\section{REFERENCES}

[1] Jayavardhana Gubbi, Rajkumar Buyya, Slaven Marusic, and Marimuthu Palaniswami. 2013. Internet of Things (IoT): A vision, architectural elements, and future directions. Future Generation Computer Systems 29, 7 (Sep 2013), 1645-1660.

[2] Lee Martin. 2015. The Promise of the Maker Movement for Education. Journal of Pre-College Engineering Education Research (J-PEER) 5, 1 (Apr 2015).

[3] Donald A Norman. 1988. The psychology of everyday things. Basic books.

[4] J J Rodríguez-Andina, M D Valdés-Peña, and M J Moure. 2015. Advanced Features and Industrial Applications of FPGAs - A Review. IEEE Transactions on Industrial Informatics 11, 4 (2015), 853-864.

[5] Evan Strasnick, Maneesh Agrawala, and Sean Follmer. 2017. Scanalog: Interactive Design and Debugging of Analog Circuits with Programmable Hardware. In Proceedings of the 30th Annual ACM Symposium on User Interface Software and Technology - UIST'17. ACM Press, New York, New York, USA, 321-330.

[6] Evan Strasnick, Sean Follmer, and Maneesh Agrawala. 2019. Pinpoint: A PCB Debugging Pipeline Using Interruptible Routing and Instrumentation. In Proceedings of the 2019 CHI Conference on Human Factors in Computing Systems. New York, New York, USA, 1-11. 